\title{
Can Optimal Rearrangement Invariant Sobolev Imbeddings be Further Extended?
}

\author{
Guillermo P. Curbera ひ. Werner J. Ricker
}

\begin{abstract}
Sobolev imbeddings (over suitable open subsets of $\mathbb{R}^{n}$ ) can be extended from the classical $L^{p}$-setting to that of more general norms (required to be rearrangement invariant) on the underlying function spaces. This has been thoroughly studied in recent years and shown to be intimately connected to an associated kernel operator (of one variable). This kernel operator always has an optimal domain (being a Banach function space, but typically not rearrangement invariant) to which it can be continuously extended. So, techniques aside, there is no a priori reason not to treat Sobolev imbeddings for non-rearrangement invariant norms. This is the aim of the present paper.
\end{abstract}

\section{Introduction And MAin RESUlts}

The aim of this note is to investigate the possibility of extending the results of [8], [9] on existence and boundedness of the optimal rearrangement invariant Sobolev imbedding to the non-rearrangement invariant setting. We formulate this more precisely.

Let $\Omega \subset \mathbb{R}^{n}(n \geq 2)$ be any bounded, open set (with Lebesgue measure one, i.e., $m(\Omega)=1$ ). The classical Sobolev inequality asserts, for $1 \leq p<n$, that there exists $C>0$ with $\|u\|_{q} \leq C\|\nabla u\|_{p}$, for every $u \in C_{0}^{1}(\Omega)$, whenever $1 \leq q \leq n p /(n-p)$, with the constant $C$ depending only on $p, q$ and $n$. Suppose that, instead of measuring $u$ in $L^{q}(\Omega)$ and the gradient $\nabla u$ in $L^{p}(\Omega)$, we consider rearrangement invariant (briefly, r.i.) Banach function spaces $X(\Omega)$ and $Y(\Omega)$ over $\Omega$ and measures $u$ in $X(\Omega)$ and $\nabla u$ in $Y(\Omega)$. Then we would be looking at an r.i. Sobolev inequality

$$
\|u\|_{X(\Omega)} \leq C\|\nabla u\|_{Y(\Omega)}, \quad u \in C_{0}^{1}(\Omega) .
$$


For $m(\Omega)=1$, the classical Sobolev inequality can be formulated as $\left\|u^{*}\right\|_{L^{q}[0,1]} \leq$ $C\left\|\left|\nabla u^{*}\right|\right\|_{L^{p}[0,1]}$, where $u^{*}$ and $|\nabla u|^{*}$ are, respectively, the decreasing rearrangements of $u$ and of the Euclidean norm of $\nabla u$. Due to Luxemburg's representation theorem, $[2,2.4 .10]$, every r.i. space on $\Omega$ is of the form $Z(\Omega):=$ $\left\{u \mid \Omega \rightarrow \mathbb{R}: u^{*} \in Z\right\}$ with $\|u\|_{Z(\Omega)}:=\left\|u^{*}\right\|_{Z}$ for some r.i. space $Z$ over $[0,1]$. Hence, the inequality (1.1) can be equivalently written as

$$
\left\|u^{*}\right\|_{X} \leq C\left\||\nabla u|^{*}\right\|_{Y}, \quad u \in C_{0}^{1}(\Omega),
$$

where now $X$ and $Y$ are r.i. Banach function spaces over $[0,1]$.

It is known that r.i. spaces $X$ and $Y$ satisfy (1.2) if and only if the kernel operator $T$ associated with Sobolev's inequality, namely

$$
T f(t):=\int_{t}^{1} f(s) s^{-1 / n^{\prime}} \mathrm{d} s, \quad t \in[0,1],
$$

is bounded from $Y$ to $X$, that is, $\|T f\|_{X} \leq K\|f\|_{Y}$, [9, Theorem 6.1]. Here, $n^{\prime}$ is the conjugate index to $n$, that is, $1 / n+1 / n^{\prime}=1$.

In [5] and [8] we considered the optimal (=maximal) r.i. domain $[T, X]^{\mathrm{ri}}$ for $T$, as given by (1.3), with $T$ taking its values in an r.i. space $X$. The maximality is to be understood in the following sense: there exists a continuous linear extension of $T$ from $[T, X]^{\text {ri }}$ into $X$ and, if $T$ has any continuous linear extension from some r.i. space $F$ (over $[0,1]$ ) into $X$, then $F$ is continuously included in $[T, X]^{\text {ri }}$. Thus, by the above mentioned result of [9], the inequality

$$
\left\|u^{*}\right\|_{X} \leq C\left\||\nabla u|^{*}\right\|_{[T, X]^{\mathrm{ri}}}, \quad u \in C_{0}^{1}(\Omega),
$$

may be considered as the optimal r.i. Sobolev inequality, since the $[T, X]^{\mathrm{ri}}$-norm cannot be replaced by any smaller r.i. norm.

Defining the Sobolev space $W_{0}^{1} Y(\Omega)$ as the completion of $C_{0}^{1}(\Omega)$ with respect to the norm $\|u\|_{W_{0}^{1} Y(\Omega)}:=\|u\|_{Y(\Omega)}+\||\nabla u|\|_{Y(\Omega)}$, it follows that (1.1) is equivalent to boundedness of the inclusion map $j: W_{0}^{1} Y(\Omega) \hookrightarrow X(\Omega)$ (for this equivalence, a generalized Poincaré inequality for r.i. spaces is needed; see [3, Lemma 4.2]). Thus, the optimality of $[T, X]^{\mathrm{ri}}$ implies that the imbedding

$$
j: W_{0}^{1}[T, X]^{\mathrm{ri}}(\Omega) \hookrightarrow X(\Omega)
$$

is bounded and optimal, that is, for the fixed range space $X(\Omega)$ we cannot replace $[T, X]^{\mathrm{ri}}(\Omega)$ by any larger r.i. space over $\Omega$. In this sense, we say that $(1.5)$ is the optimal r.i. Sobolev imbedding and $W_{0}^{1}[T, X]^{\mathrm{ri}}(\Omega)$ is the optimal r.i. Sobolev domain corresponding to the fixed range space $X(\Omega)$. However, the methods of [8] aside, there is no a priori reason to remain within the r.i. setting for the optimal Sobolev imbedding, provided one can "make sense" of the more general setting. This is indeed possible as we now explain. 
The kernel operator $T$ can always be extended further, with $T$ still taking its values in $X$, to a Banach function space (briefly, B.f.s.) which is larger (in general) than $[T, X]^{\mathrm{ri}}$. This larger space is the optimal (= maximal) B.f.s. domain for $T$ and is denoted by $[T, X]$. The maximality is, again, to be understood as above: there exists a continuous linear extension of $T$ from $[T, X]$ into $X$ and, if $T$ has any continuous linear extension from some B.f.s. $F$ (over $[0,1]$ ) into $X$, then $F$ is continuously included in $[T, X]$. Of course, if the inclusion $[T, X]^{\mathrm{ri}} \hookrightarrow[T, X]$ is proper, then $[T, X]$ cannot be r.i. However, $[T, X]^{\mathrm{ri}}=[T, X]$ occurs only for $X=L^{n^{\prime}, 1}([0,1]),[8$, Theorem 2.4], that is, for "almost every" r.i. space $X$ we have $[T, X]^{\mathrm{ri}} \subsetneq[T, X]$. Accordingly, for $X \neq L^{n^{\prime}, 1}([0,1])$ there is scope for replacing the r.i. space $[T, X]^{\mathrm{ri}}$ with the larger non-r.i. B.f.s. $[T, X]$. We would then expect to obtain an optimal Sobolev inequality typically more general than (1.4), namely

$$
\left\|u^{*}\right\|_{X} \leq C\left\||\nabla u|^{*}\right\|_{[T, X]}, \quad u \in C_{0}^{1}(\Omega),
$$

and also an optimal Sobolev imbedding more general than (1.5), namely

$$
j: W_{0}^{1}[T, X](\Omega) \hookrightarrow X(\Omega) .
$$

However, difficulties arise when one attempts to carry out this procedure, that is, to replace $[T, X]^{\mathrm{ri}}$ with $[T, X]$.

Firstly, potential difficulties appear due to the fact that $[T, X]$ is a B.f.s. which, as pointed out above, is in general not r.i. Thus, in this case, it is unclear how the spaces $[T, X](\Omega)$ and hence, also $W_{0}^{1}[T, X](\Omega)$, should even be defined, let alone what properties they may have. This question is answered in Section 2 where, due to specific properties of the kernel operator $T$ and of the particular B.f.s. $[T, X]$, we show that $[T, X](\Omega)$ is always an r.i. quasi-Banach function space and hence, that $W_{0}^{1}[T, X](\Omega)$ is always a quasi-Banach space (containing $W_{0}^{1}[T, X]^{\mathrm{ri}}(\Omega)$ ).

Secondly, even though $W_{0}^{1}[T, X](\Omega)$ always exists, it is unclear whether the Sobolev imbedding (1.7) exists or not. In this regard, we show that the imbedding (1.7) fails for a (large) class of r.i. spaces $X$ which satisfy a relatively "simple constraint" (see (1.8) below) on their fundamental function $\varphi_{X}$ (defined by $\left.\varphi_{X}(t):=\left\|\chi_{[0, t]}\right\|_{X}\right)$.

Theorem 1.1. Let $X$ be an r.i. space over $[0,1]$ satisfying

$$
\lim _{t \rightarrow 0} \varphi_{X}(t) / t^{1 / n^{\prime}}=0 .
$$

Then the optimal Sobolev imbedding (1.7) fails to exist for the space X.

Recall that the r.i. space $X=L^{p, q}([0,1])$, for $1 \leq p, q \leq \infty$ with $(p, q) \neq$ $(1, \infty)$, is defined as the set of functions $f:[0,1] \rightarrow \mathbb{R}$ for which

$$
\begin{aligned}
& \|f\|_{p, q}:=\left(\int_{0}^{1}\left(t^{1 / p} f^{*}(t)\right)^{q} \frac{\mathrm{d} t}{t}\right)^{1 / q}, \quad q<\infty, \\
& \|f\|_{p, \infty}:=\sup _{0<t \leq 1} t^{1 / p} f^{*}(t),
\end{aligned}
$$


is finite. For these spaces, Theorem 1.1 implies that the optimal Sobolev imbedding (1.7) fails to exist whenever $1 \leq p<n^{\prime}$ and $1 \leq q \leq \infty$. In particular, this is the case for $X=L^{p}([0,1])$ with $1 \leq p<n^{\prime}$.

Other relevant families of classical r.i. spaces are the Lorentz and Marcinkiewicz spaces. Let $\varphi$ be an increasing, concave function on $[0,1]$ with $\varphi(0)=0$. The Lorentz space $\Lambda_{\varphi}$ associated to $\varphi$ is defined by

$$
\Lambda_{\varphi}:=\left\{f:\|f\|_{\Lambda_{\varphi}}:=\int_{0}^{1} f^{*}(s) \mathrm{d} \varphi(s)<\infty\right\},
$$

and the Marcinkiewicz space $M_{\varphi}$ associated to $\varphi$ is given by

$$
M_{\varphi}:=\left\{f:\|f\|_{M_{\varphi}}:=\sup _{0<t \leq 1} \frac{\varphi(t)}{t} \int_{0}^{t} f^{*}(s) \mathrm{d} s<\infty\right\} .
$$

Then, Theorem 1.1 implies that the optimal Sobolev imbedding (1.7) fails to exist, for $X$ being $\Lambda_{\varphi}$ or $M_{\varphi}$, whenever $\lim _{t \rightarrow 0} \varphi(t) / t^{1 / n^{\prime}}=0$. For example, this is the case for the classical Zygmund space $L \log L([0,1])$.

Observe that spaces $X$ satisfying condition (1.8) also satisfy the inclusion $L^{n^{\prime}, \infty}([0,1]) \subsetneq X$.

In the other direction, for what r.i. spaces $X$ does the optimal Sobolev imbedding (1.7) exist and, in the event that it does, is it a genuine extension of (1.5) or not? An answer for certain classes of r.i. spaces $X$ is given by the following result.

Theorem 1.2. Let $X=\Lambda_{\varphi}$ be a Lorentz $\Lambda$-space such that $\varphi(t) / t^{1 / n^{\prime}}$ is equivalent to a decreasing function. Then the optimal Sobolev imbedding (1.7) exists for $X=\Lambda_{\varphi}$ but, it is not a further extension of the optimal r.i. Sobolev imbedding (1.5).

Theorem 1.2 applies to $X=L^{\infty}([0,1])$, since this space can be viewed as a Lorentz $\Lambda_{\varphi}$-space for $\varphi(t)=\chi_{(0,1]}$. Its norm, arising from the Riemann-Stieljes integral $\|f\|_{\Lambda_{\varphi}}=\int_{0}^{1} f^{*}(s) \mathrm{d} \varphi(s)=f^{*}(0)+\int_{0}^{1} f^{*}(s) \varphi^{\prime}(s) \mathrm{d} s$, is then $\|f\|_{\Lambda_{\varphi}}=$ $f^{*}(0)=\|f\|_{\infty}$.

Note that spaces $X=\Lambda_{\varphi}$ satisfying the assumption of Theorem 1.2 also satisfy $\Lambda_{\varphi} \subseteq L^{n^{\prime}, 1}([0,1])$.

Another criterion can be formulated in terms of the lower and upper Boyd indices, respectively $\underline{\alpha}_{X}$ and $\bar{\alpha}_{X}$, of the r.i. space $X$; see $[2,3.5 .12]$ for the definition.

Theorem 1.3. Let $X$ be an r.i. space over $[0,1]$ whose Boyd indices satisfy

$$
0<\underline{\alpha}_{X} \leq \bar{\alpha}_{X}<\frac{1}{n^{\prime}} .
$$

Then the optimal Sobolev imbedding (1.7) exists for X but, it is not a further extension of the optimal r.i. Sobolev imbedding (1.5). 
The requirement of Theorem 1.3 holds for all spaces $X=L^{p, q}([0,1])$ with $n^{\prime}<p<\infty$ and $1 \leq q \leq \infty$. In particular, the conclusion of the theorem holds for all $X=L^{p}([0,1])$ with $n^{\prime}<p<\infty$, for all spaces $X=L^{p, 1}([0,1])$ with $n^{\prime}<p<\infty$ (which also follows from Theorem 1.2), and for the weak- $L^{p}$ spaces $X=L^{n^{\prime}, \infty}([0,1])$ with $n^{\prime}<p<\infty$.

In Theorems 1.2 and 1.3 one may ask why the imbedding (1.7) is not a genuine extension of (1.5)? The answer is that the Sobolev spaces $W_{0}^{1}[T, X](\Omega)$ and $W_{0}^{1}[T, X]^{\mathrm{ri}}(\Omega)$ turn out to be isomorphic (via the identity operator), that is, $W_{0}^{1}[T, X](\Omega)$ is obtained by renorming $W_{0}^{1}[T, X]^{\mathrm{ri}}(\Omega)$. This is due to the fact that $[T, X](\Omega)$ and $[T, X]^{\mathrm{ri}}(\Omega)$ are isomorphic (also via the identity operator), a somewhat surprising fact since $[T, X]$ and $[T, X]^{\mathrm{ri}}$ only coincide for the special case of $X=L^{n^{\prime}, 1}([0,1])$. The reason for $[T, X](\Omega)$ and $[T, X]^{\mathrm{ri}}(\Omega)$ being isomorphic is that the cone of nonnegative, decreasing functions in $[T, X]$, which we denote by $[T, X]_{\mathrm{d}}^{+}$, turns out (under certain conditions on $X$ ) to be a subset of $[T, X]^{\mathrm{ri}}$; see Proposition 4.2.

\section{Preliminary Results}

In this section we collect some basic notation and concepts needed in the sequel, define precisely the optimal domain $[T, X]$, and establish the existence and various properties of the spaces $[T, X](\Omega)$ and $W_{0}^{1}[T, X](\Omega)$.

2.1 Let $\Omega \subseteq \mathbb{R}^{n}$ be a bounded, open set (with $m(\Omega)=1$ for normalization purposes). We denote by $\mathcal{M}$ the space of all (classes of) Lebesgue measurable, a.e. finite functions defined in $\Omega$. A quasi-Banach function space $Z$ over $\Omega$ (briefly, q-B.f.s.) is a linear space $Z \subseteq \mathcal{M}$ equipped with a quasi-norm (i.e., a [0, $\infty$ )-valued functional $\|\cdot\|$ defined on $Z$ for which $\|f\|=0$ implies $f=0$, for each $\lambda \in \mathbb{R}$ and $f \in Z$ we have $\|\lambda f\|=|\lambda| \cdot\|f\|$, and there exists a constant $C>0$ such that $\|f+g\| \leq C(\|f\|+\|g\|)$ for all $f, g \in Z)$ and satisfying the ideal property, that is, $g \in Z$ and $\|g\| \leq\|f\|$ whenever $f \in Z$ and $|g| \leq|f|$ a.e. If $\|\cdot\|$ is actually subadditive, that is, $\|f+g\| \leq\|f\|+\|g\|$ for all $f, g \in Z$, then $Z$ is a B.f.s.

The distribution function of $f \in \mathcal{M}$ is defined by $m_{f}(\lambda):=m(\{x \in$ $\Omega:|f(x)|>\lambda\})$ for $\lambda \in[0, \infty)$. Functions $g$ and $f$ are said to be equimeasurable if $m_{g}(\lambda)=m_{f}(\lambda)$, for every $\lambda>0$. A q-B.f.s. $Z$ is said to be rearrangement invariant if $g \in Z$ and $\|g\|=\|f\|$ whenever $f \in Z$ and $f$ and $g$ are equimeasurable, and if $Z$ satisfies the Fatou property, that is, $0 \leq f_{k} \uparrow$ with $\left\{f_{k}\right\} \subset Z$ and $\sup _{k}\left\|f_{k}\right\|<\infty$ implies $f:=\sup _{k} f_{k} \in Z$ and $\left\|f_{k}\right\| \uparrow\|f\|$. If $Z$ is a B.f.s. which is r.i., then we simply say that $Z$ is an r.i. space. In this case, $L^{\infty}(\Omega) \subseteq Z \subseteq L^{1}(\Omega)$ continuously.

The decreasing rearrangement of $f: \Omega \rightarrow \mathbb{R}$ is the function $f^{*}:[0,1] \rightarrow \mathbb{R}^{+}$ which is the right continuous inverse of its distribution function. Let $Z$ be an r.i. q-B.f.s. over $[0,1]$ and $f \in Z$. Then also $f^{*} \in Z$ and $\left\|f^{*}\right\|=\|f\|$. The dilation operator $D_{\alpha}$ for $\alpha>0$ is given by $f(t) \mapsto f(\alpha t) \cdot \chi_{[0, \gamma]}(t)$ where $\gamma=\min \{1,1 / \alpha\}$. Dilation operators are bounded on all r.i. spaces $Z$ over $[0,1]$. 
We will need several properties of decreasing rearrangements. Let $f$ and $g$ be measurable functions. We write $g \prec f$ whenever

$$
\int_{0}^{t} g^{*}(s) \mathrm{d} s \leq \int_{0}^{t} f^{*}(s) \mathrm{d} s, \quad t>0 .
$$

For arbitrary nonnegative, measurable functions $f, g$, we always have

$$
\int f g \leq \int f^{*} g^{*} .
$$

We will repeatedly make use of Hardy's lemma:

Lemma 2.1 (Hardy's Lemma). Let $\varphi$ and $\psi$ be nonnegative, measurable functions on $(0, \infty)$ satisfying

$$
\int_{0}^{t} \varphi(s) \mathrm{d} s \leq \int_{0}^{t} \psi(s) \mathrm{d} s, \quad t>0 .
$$

Then, for every nonnegative, decreasing function $h$ on $(0, \infty)$, we have

$$
\int_{0}^{\infty} \varphi(s) h(s) \mathrm{d} s \leq \int_{0}^{\infty} \psi(s) h(s) \mathrm{d} s .
$$

For these facts (and others) concerning r.i. spaces, see [2] and [12].

2.2 We now consider the optimal lattice domain for $T$, with $T$ given via (1.3) and considered as taking its values in a given r.i. space $X$ over $[0,1]$. Let $\mathcal{M}^{+}$consist of those elements of $\mathcal{M}$ which are $[0, \infty)$-valued a.e. . For $f \in \mathcal{M}^{+}$, note that $(T f)(x) \in[0, \infty]$. Let $\mathcal{M}_{T}:=\left\{f \in \mathcal{M}|T| f \mid \in \mathcal{M}^{+}\right\}$; it is an ideal (i.e., a linear subspace with the ideal property) in $\mathcal{M}$ and $T: \mathcal{M}_{T} \rightarrow \mathcal{M}$ defines a linear and positive map. We have that $L^{\infty}([0,1]) \subseteq \mathcal{M}_{T}$ and $T\left(L^{\infty}([0,1])\right) \subseteq L^{\infty}([0,1])$. Moreover, $T: L^{\infty}([0,1]) \rightarrow L^{\infty}([0,1])$ is continuous. Since $L^{\infty}([0,1])$ is continuously included in $X$, it follows that $T: L^{\infty}([0,1]) \rightarrow X$ continuously. The question arises: which is the largest ideal in $\mathcal{M}_{T}$ which $T$ maps into $X$ ? The answer is (obviously)

$$
[T, X]:=\left\{f \in \mathcal{M}_{T}: T|f| \in X\right\} .
$$

If we define $\|f\|_{[T, X]}:=\|T|f|\|_{X}$, for $f \in[T, X]$, then $[T, X]$ becomes a B.f.s. with the property that $T:[T, X] \rightarrow X$ is continuous (with norm one). Moreover, if $Y$ is any B.f.s. (relative to $\mathcal{M}$ ) such that $T: Y \rightarrow X$ is continuous, then $T|f| \in$ $X$, for $f \in Y$, and $\|T|f|\|_{X} \leq C\|f\|_{Y}$, for $f \in Y$, that is, $Y \subseteq[T, X]$ and $\|f\|_{[T, X]} \leq C\|f\|_{Y}$, for $f \in Y$. Hence, $Y$ is continuously included in $[T, X]$. In this sense we say that $[T, X]$ is the optimal lattice domain for $T$, with $T$ taking values in $X$. See [4], [5], [7] for various aspects of such spaces. 
2.3 Given $\Omega \subset \mathbb{R}^{n}$ (bounded, open and with $m(\Omega)=1$ ) and an r.i. space $X$ over $[0,1]$, we define

$$
[T, X](\Omega):=\left\{u: \Omega \rightarrow \mathbb{R} \mid T u^{*} \in X\right\},
$$

equipped with the functional

$$
\|u\|_{[T, X](\Omega)}:=\left\|T u^{*}\right\|_{X}=\left\|u^{*}\right\|_{[T, X]}, \quad u \in[T, X](\Omega) .
$$

For example, if $X=L^{1}([0,1])$, then $\left[T, L^{1}\right]=L^{1}\left(t^{1 / n} \mathrm{~d} t\right)$, [5, Corollary 4.3], and so

$$
\left[T, L^{1}\right](\Omega)=\left\{u: \Omega \rightarrow \mathbb{R}:\|u\|_{\left[T, L^{1}\right](\Omega)}=\int_{0}^{1} u^{*}(t) t^{1 / n} \mathrm{~d} t<\infty\right\} .
$$

Note that $\|\cdot\|_{\left[T, L^{1}\right](\Omega)}$ is not a norm.

Proposition 2.2. Let $X$ be an r.i. space over $[0,1]$. Then $[T, X](\Omega)$ is always an r.i. quasi-Banach function space.

Proof. All properties are standard to verify except for: closedness under addition (and subadditivity of the functional (2.2)), the Fatou property, and completeness.

The proof that $[T, X](\Omega)$ is closed under addition is more transparent when written for a general kernel operator

$$
T_{K} f(x):=\int_{0}^{1} f(y) K(x, y) \mathrm{d} y
$$

for $x \in[0,1]$, with $K \geq 0$ satisfying the property

$$
K(x, 2 y) \leq C K\left(\frac{x}{2}, y\right), \quad(x, y) \in[0,1] \times\left[0, \frac{1}{2}\right]
$$

for some $C>0$. For $T$ as in (1.3) this is the case with $C=2^{1 / n^{\prime}}$ and $K(x, y)=$ $y^{-1 / n^{\prime}} \chi_{[x, 1]}(y)$. Let $u, v \in[T, X](\Omega)$. Taking into account the inequality

$$
(u+v)^{*}(y) \leq u^{*}\left(\frac{y}{2}\right)+v^{*}\left(\frac{y}{2}\right),
$$


$[2,2.1 .7]$, we have

$$
\begin{aligned}
T(u+v)^{*}(x) & =\int_{0}^{1} K(x, y)(u+v)^{*}(y) \mathrm{d} y \\
& \leq \int_{0}^{1} K(x, y) u^{*}\left(\frac{y}{2}\right) \mathrm{d} y+\int_{0}^{1} K(x, y) v^{*}\left(\frac{y}{2}\right) \mathrm{d} y \\
& =2 \int_{0}^{1 / 2} K(x, 2 s) u^{*}(s) \mathrm{d} s+2 \int_{0}^{1 / 2} K(x, 2 s) v^{*}(s) \mathrm{d} s \\
& \leq 2 C \int_{0}^{1} K\left(\frac{x}{2}, s\right) u^{*}(s) \mathrm{d} s+2 C \int_{0}^{1} K\left(\frac{x}{2}, s\right) v^{*}(s) \mathrm{d} s \\
& =2 C\left(T u^{*}\left(\frac{x}{2}\right)+T v^{*}\left(\frac{x}{2}\right)\right) \\
& =2 C\left(D_{1 / 2}\left(T u^{*}\right)(x)+D_{1 / 2}\left(T v^{*}\right)(x)\right) .
\end{aligned}
$$

Since $X$ is r.i., $D_{1 / 2}$ maps $X$ boundedly into $X$. From $T u^{*}, T v^{*} \in X$, we conclude that both $D_{1 / 2} T u^{*}, D_{1 / 2} T v^{*} \in X$. Thus, $T(u+v)^{*} \in X$ (i.e., $u+v \in$ $[T, X](\Omega))$ and

$$
\begin{aligned}
\|u+v\|_{[T, X](\Omega)} & =\left\|T(u+v)^{*}\right\|_{X} \\
& \leq 2 C\left\|D_{1 / 2}\right\|\left(\left\|T u^{*}\right\|_{X}+\left\|T v^{*}\right\|_{X}\right) \\
& =2 C\left\|D_{1 / 2}\right\|\left(\|u\|_{[T, X](\Omega)}+\|v\|_{[T, X](\Omega)}\right) .
\end{aligned}
$$

Accordingly, $[T, X](\Omega)$ is a linear space and (2.2) is a quasi-norm.

To see that $[T, X](\Omega)$ has the Fatou property let $0 \leq u_{k} \uparrow$ with $\left\{u_{k}\right\} \subseteq$ $[T, X](\Omega)$ and $\sup _{k}\left\|u_{k}\right\|_{[T, X](\Omega)}<\infty$. Then $0 \leq u_{k}^{*} \uparrow$ with $\left\{u_{k}^{*}\right\} \subseteq[T, X]$ and $\sup _{k}\left\|u_{k}^{*}\right\|_{[T, X]}<\infty$. Since $X$ has the Fatou property, so does $[T, X]$, [7, Proposition 3.1(ii)]. Thus, there exists $g:=\sup u_{k}^{*} \in[T, X]$ with $\left\|u_{k}^{*}\right\|_{[T, X]} \uparrow$ $\|g\|_{[T, X]}$. Set $u:=\sup _{k} u_{k}$. Then $u^{*}=g \in[T, X]$ and so $u \in[T, X](\Omega)$ with $\left\|u_{k}\right\|_{[T, X](\Omega)}=\left\|u_{k}^{*}\right\|_{[T, X]} \uparrow\left\|u^{*}\right\|_{[T, X]}=\|u\|_{[T, X](\Omega)}$. That is, $[T, X](\Omega)$ has the Fatou property.

Establishing completeness of the quasi-normed function space $[T, X](\Omega)$ requires some care, since the quasi-norm need not be countably subadditive. From a result of Aoki and Rolewicz, [10, Theorem 1.3], it follows that there exists $p \in(0,1]$ such that, for every $k \geq 1$, we have

$$
\left\|\sum_{1}^{k} f_{i}\right\|_{[T, X](\Omega)}^{p} \leq 4 \sum_{1}^{k}\left\|f_{i}\right\|_{[T, X](\Omega)}^{p}, \quad f_{i} \in[T, X](\Omega) .
$$


This inequality and the Fatou property of $[T, X](\Omega)$ imply the Riesz-Fischer property for $[T, X](\Omega)$. Namely, whenever $\left\{f_{k}\right\} \subseteq[T, X](\Omega)^{+}$satisfy

$$
\sum_{1}^{\infty}\left\|f_{k}\right\|_{[T, X](\Omega)}^{p}<\infty
$$

then $\sum_{1}^{\infty} f_{k} \in[T, X](\Omega)$ and

$$
\left\|\sum_{1}^{\infty} f_{k}\right\|_{[T, X](\Omega)}^{p} \leq 4 \sum_{1}^{\infty}\left\|f_{k}\right\|_{[T, X](\Omega)}^{p} .
$$

From here the proof of completeness follows the classical lines; see [14, pp. 444445], for example.

Defining the generalized Sobolev space $W_{0}^{1}[T, X](\Omega)$ as the completion of $C_{0}^{1}(\Omega)$ with respect to the quasi-norm

$$
\|u\|_{W_{0}^{1}[T, X](\Omega)}:=\|u\|_{[T, X](\Omega)}+\||\nabla u|\|_{[T, X](\Omega),},
$$

we obtain a quasi-Banach space.

\section{Non EXistence of the Sobolev Imbedding}

In this section we establish Theorem 1.1. First, a useful inequality.

Proposition 3.1. Let $X$ be an r.i. space over [0,1] for which the optimal Sobolev imbedding (1.7) exists. Then the fundamental function $\varphi_{X}$ of $X$ satisfies, for some constant $K>0$,

$$
\varphi_{X}\left(x_{2}\right) \leq K\left(\left(x_{1}+x_{2}\right)^{1 / n} \varphi_{X}\left(x_{1}+x_{2}\right)+\frac{\varphi_{X}\left(x_{1}\right)}{x_{1}^{1 / n^{\prime}}}\right),
$$

for $0<x_{1}<x_{2}$ with $\left(x_{1}+x_{2}\right)<1$.

Proof. Since an arbitrary open set in $\mathbb{R}^{n}$ always contains an open ball, a scaling and translation argument shows that we may assume $\Omega \subset \mathbb{R}^{n}$ is the open ball centered at the origin with $m(\Omega)=1$. In this case, $|x|<1 / C_{n}^{1 / n}<1$ for each $x \in \Omega$, where $C_{n}$ is the measure of the Euclidean unit ball in $\mathbb{R}^{n}$.

Fix $0<x_{1}<x_{2}$ with $\left(x_{1}+x_{2}\right)<1$. Suppose there exists a function $u \in$ $W_{0}^{1}[T, X](\Omega)$ such that

(i) $\chi_{\left[0, x_{2}\right]} \leq u^{*} \leq X_{\left[0, x_{2}+x_{1}\right]}$,

(ii) $|\nabla u|^{*} \leq\left(M / x_{1}\right) \chi_{\left[0, x_{1}\right]}$, for some constant $M>0$. 
Then, from (ii), we have $T|\nabla u|^{*} \leq\left(M / x_{1}\right) T \chi_{\left[0, x_{1}\right]}$. For $0 \leq \alpha \leq 1$, it is routine to check that

$$
T\left(\chi_{[0, \alpha]}\right)(t) \leq n \alpha^{1 / n} \chi_{[0, \alpha]}(t) \quad t \in[0,1] .
$$

Applying (3.2), we deduce that $T|\nabla u|^{*} \leq n M\left(x_{1}^{1 / n} / x_{1}\right) \chi_{\left[0, x_{1}\right]}$. In a similar way, from (i), we have $T u^{*} \leq n\left(x_{1}+x_{2}\right)^{1 / n} \chi_{\left[0, x_{1}+x_{2}\right]}$.

According to (1.7), applied to this function $u$ we get, for some $C>0$,

$$
\begin{aligned}
\varphi_{X}\left(x_{2}\right) & =\left\|\chi_{\left[0, x_{2}\right]}\right\|_{X} \leq\left\|u^{*}\right\|_{X}=\|u\|_{X(\Omega)} \\
& \leq C\left(\|u\|_{[T, X](\Omega)}+\||\nabla u|\|_{[T, X](\Omega)}\right)=C\left(\left\|T u^{*}\right\|_{X}+\left\|T|\nabla u|^{*}\right\|_{X}\right) \\
& \leq n C\left(x_{1}+x_{2}\right)^{1 / n}\left\|\chi_{\left[0, x_{1}+x_{2}\right]}\right\|_{X}+n C M\left\|x_{\left[0, x_{1}\right]}\right\|_{X} / x_{1}^{1 / n^{\prime}} \\
& \leq K\left(\left(x_{1}+x_{2}\right)^{1 / n} \varphi_{X}\left(x_{1}+x_{2}\right)+\varphi_{X}\left(x_{1}\right) / x_{1}^{1 / n^{\prime}}\right) .
\end{aligned}
$$

This proves the result, modulo the existence of $u$.

Now, define $u(x):=g(|x|)$, for $x \in \Omega$ where, for $0<a<b<1 / C_{n}^{1 / n}$ fixed, $g$ is the continuous function on $[0,1]$ which equals one on $[0, a]$, zero on $[b, 1]$, and is affine on $[a, b]$. Note that $g$ is bounded, nonnegative, decreasing and differentiable at every point $t \neq a, b$. Then $u$ is radial and $u^{*}(t)=$ $g\left(\left(t / C_{n}\right)^{1 / n}\right)$. Setting $a:=\left(x_{2} / C_{n}\right)^{1 / n}$ and $b:=\left(\left(x_{2}+x_{1}\right) / C_{n}\right)^{1 / n}$, we have that $u^{*}$ is the continuous function which equals one on $\left[0, x_{2}\right]$, zero on $\left[x_{2}+\right.$ $\left.x_{1}, 1\right]$, and is linear on $\left[x_{2}, x_{2}+x_{1}\right]$. Hence, (i) is satisfied.

We now turn to (ii). Direct computation shows that $|\nabla u(x)|=\left|g^{\prime}(|x|)\right|$, for $|x| \neq a, b$. Observe that $\left|g^{\prime}(t)\right|=(1 /(b-a)) \chi_{[a, b]}(t)$, for $t \neq a, b$. In order to determine $|\nabla u|^{*}$ we need to take into account that $\left|g^{\prime}\right|$ is not a decreasing function. Doing so, it turns out that $|\nabla u|^{*}=(1 /(b-a)) \chi_{[0, \alpha]}$, where $\alpha:=m\left(\left\{x \in \mathbb{R}^{n}|a \leq| x \mid<b\right\}\right)$. For the above choice of $a=\left(x_{2} / C_{n}\right)^{1 / n}$ and $b=\left(\left(x_{2}+x_{1}\right) / C_{n}\right)^{1 / n}$, we obtain $\alpha=x_{1}$. Estimating $1 /(b-a)$, via the mean value theorem for example, we get $1 /(b-a) \leq M / x_{1}$. This establishes (ii).

Proof of Theorem 1.1. If (1.7) did hold for $X$, then setting $x_{2}>0$ and taking the limit in (3.1) for $x_{1} \rightarrow 0$ we get $1 \leq K x_{2}^{1 / n}$, which cannot hold for every $x_{2}>0$.

We end this section with a result which shows how condition (1.8), for an r.i. space $X$, can strongly influence the geometry of the space $[T, X](\Omega)$ and hence, also of $W_{0}^{1}[T, X](\Omega)$.

Proposition 3.2. Let $X$ be an r.i. space over $[0,1]$ such that $\varphi_{X}$ satisfies (1.8), that is, $\lim _{t \rightarrow 0} \varphi_{X}(t) / t^{1 / n^{\prime}}=0$. Then the q-B.f.s. $[T, X](\Omega)$ is not normable.

Proof. We will deduce that $[T, X](\Omega)$ is not normable by appealing to the classical theorem of Kolmogorov; see [11, Section 15.10(1)]. Namely, we show 
that the convex hull of every neighbourhood $\mathcal{U}$ of the origin is unbounded. Since the topology of $[T, X](\Omega)$ is generated by a quasi-norm, it suffices to prove this just for the unit ball

$$
\mathcal{U}_{1}=\left\{u \in[T, X](\Omega):\|u\|_{[T, X](\Omega)} \leq 1\right\} .
$$

Given any $k \geq 1$, let $\left(\Omega_{i}\right)_{1}^{k}$ be a partition of $\Omega$ into sets of equal measure. Setting $u_{i}=\chi_{\Omega_{i}}$, for $1 \leq i \leq k$, we have $\left(u_{i}\right)_{1}^{k} \subset[T, X](\Omega)$ with $u_{i}^{*}=\chi_{[0,1 / k]}$ and $\left(\sum_{1}^{k} u_{i}\right)^{*}=\chi_{[0,1]}$. Thus, setting $\alpha_{i}=1 / k$, for $1 \leq i \leq k$, we have by (3.2) that

$$
\begin{aligned}
\frac{\left\|\sum_{1}^{k} \alpha_{i} u_{i}\right\|_{[T, X](\Omega)}}{\left\|u_{i}\right\|_{[T, X](\Omega)}} & =\frac{\left\|T\left(\sum_{1}^{k} \alpha_{i} u_{i}\right)^{*}\right\|_{X}}{\left\|T u_{i}^{*}\right\|_{X}} \\
& =\frac{(1 / k)\left\|T \chi_{[0,1]}\right\|_{X}}{\left\|T \chi_{[0,1 / k]}\right\|_{X}} \\
& \geq \frac{\left\|T \chi_{[0,1]}\right\|_{X}}{k n(1 / k)^{1 / n}\left\|\chi_{[0,1 / k]}\right\|_{X}} \\
& =\frac{\left\|T \chi_{[0,1]}\right\|_{X} / n}{\frac{\varphi_{X}(1 / k)}{(1 / k)^{1 / n^{\prime}}}}
\end{aligned}
$$

which, by (1.8), goes to infinity as $k \rightarrow \infty$ (since $\left\|T \chi_{[0,1]}\right\|_{X} / n$ is constant).

Remark 3.3. If $X$ is an r.i. space satisfying the condition of Proposition 3.2 (i.e., (1.8)) and, additionally, is order continuous (i.e., order bounded, increasing sequences in $X$ are norm convergent), then the q-B.f.s. $[T, X](\Omega)$ has a trivial dual space. This follows from the fact that the convex hull of the unit ball of $[T, X](\Omega)$ coincides with $[T, X](\Omega)$; see $[11$, Section $17.4(1)]$. To see this, let $u \in[T, X](\Omega)$. For $M>0$ and $k \geq 1$, let $\Omega_{0}=\{x \in \Omega:|u(x)|>M\}$ and $\left(\Omega_{i}\right)_{1}^{k}$ be a partition of $\Omega \backslash \Omega_{0}$ into sets of equal measure. Then

$$
u=\frac{1}{2}\left(2 u \chi_{\Omega_{0}}\right)+\sum_{i=1}^{k} \frac{1}{2 k}\left(2 k u \chi_{\Omega_{i}}\right) .
$$

Since $X$ is order continuous, so is $[T, X]$, [7, Proposition 3.1(i)]. An argument similar to that in the proof of Proposition 2.2 for the Fatou property, then establishes that $[T, X](\Omega)$ is order continuous. This implies that

$$
\lim _{M \rightarrow \infty}\left\|u \chi_{\Omega_{0}}\right\|_{[T, X](\Omega)}=0
$$


see $[2,1.3 .5]$. Thus, $\left\|2 u \chi_{\Omega_{0}}\right\|_{[T, X](\Omega)} \leq 1$ for an appropriate $M>0$. Fix $1 \leq i \leq$ $k$. Since $m\left(\Omega_{i}\right) \leq 1 / k$ we have, via (3.2), that

$$
\begin{aligned}
\left\|2 k u \chi_{\Omega_{i}}\right\|_{[T, X](\Omega)} & \leq 2 k M\left\|\chi_{\Omega_{i}}\right\|_{[T, X](\Omega)} \leq 2 k M\left\|\chi_{[0,1 / k]}\right\|_{[T, X]} \\
& =2 k M\left\|T \chi_{[0,1 / k]}\right\|_{X} \leq \frac{2 k M n}{k^{1 / n}}\left\|\chi_{[0,1 / k]}\right\|_{X} \\
& =2 M n \frac{\varphi_{X}(1 / k)}{(1 / k)^{1 / n^{\prime}}}
\end{aligned}
$$

which, by (1.8), can be made smaller than one, for a suitable choice of $k$. This result applies, for example, to all spaces $X=L^{p, q}([0,1])$ with $1 \leq p<n^{\prime}$ and $q \neq \infty$.

\section{EXISTENCE OF THE EXTENDED SoboleV Imbedding}

In this section we prove and discuss Theorems 1.2 and 1.3. Recall that $[T, X]_{\mathrm{d}}^{+}$ denotes the cone of nonnegative, decreasing functions in $[T, X]$.

Dilation operators are always bounded on r.i. spaces, but not necessarily on a general B.f.s. Nevertheless, as we now show, they are always bounded on spaces of the type $[T, X]$. For a B.f.s. $Y$, the operator norms of continuous dilation operators $D_{\alpha}$ on $Y$ are denoted by $\left\|D_{\alpha}\right\|_{Y}$.

Proposition 4.1. Let $X$ be an r.i. space over $[0,1]$. For each $\alpha>0$, the dilation operator $D_{\alpha}$ is bounded on $[T, X]$ and

$$
\left\|D_{\alpha}\right\|_{[T, X]} \leq \frac{\left\|D_{\alpha}\right\|_{X}}{\alpha^{1 / n}} .
$$

Proof. Let $\alpha \geq 1$. Direct computation shows that $T D_{\alpha} f=\left(1 / \alpha^{1 / n}\right) D_{\alpha} T f$. Since $T f \in X$ whenever $f \in[T, X]$ and $D_{\alpha}$ maps the r.i. space $X$ into itself, we can conclude from the previous equality that $D_{\alpha}$ maps $[T, X]$ into itself. Via the definition of the norm in $[T, X]$ and the identity $\left|D_{\alpha} f\right|=D_{\alpha}|f|$, it is routine to establish (4.1).

In the case $0<\alpha<1$, for $f \geq 0$ we have $0 \leq T D_{\alpha} f \leq\left(1 / \alpha^{1 / n}\right) D_{\alpha} T f$. Using these inequalities and the fact that $[T, X]$ is a lattice, it is possible to argue analogously as for $\alpha \geq 1$.

Proposition 4.2. Let $X$ be an r.i. space over $[0,1]$. The quasi-Banach function space $[T, X](\Omega)$ and the r.i. Banach function space $[T, X]^{\mathrm{ri}}(\Omega)$ are isomorphic (via the identity operator) if and only if $[T, X]_{\mathrm{d}}^{+} \subset[T, X]^{\mathrm{ri}}$ and there exists a constant $C>0$ such that

$$
\|f\|_{[T, X]^{\mathrm{ri}}} \leq C\|f\|_{[T, X]}, \quad f \in[T, X]_{\mathrm{d}}^{+} .
$$


Proof. We first prove necessity. Since always $[T, X]^{\mathrm{ri}} \subset[T, X]$ continuously, we have $[T, X]^{\mathrm{ri}}(\Omega) \subset[T, X](\Omega)$ and $K\|u\|_{[T, X](\Omega)} \leq\|u\|_{[T, X]^{\mathrm{ri}}(\Omega)}$, for every $u \in[T, X]^{\mathrm{ri}}(\Omega)$ and some constant $K>0$. Let $u \in[T, X](\Omega)$. Then $u^{*} \in[T, X]$ with $u^{*}$ decreasing and so $u^{*} \in[T, X]_{\mathrm{d}}^{+}$. From the assumption $[T, X]_{\mathrm{d}}^{+} \subset[T, X]^{\mathrm{ri}}$, it follows that $u^{*} \in[T, X]^{\mathrm{ri}}$, that is, $u \in[T, X]^{\mathrm{ri}}(\Omega)$. Then, according to (4.2), we have $\left\|u^{*}\right\|_{[T, X]^{\mathrm{ri}}} \leq C\left\|u^{*}\right\|_{[T, X]}$, that is, $\|u\|_{[T, X]^{\mathrm{ri}}(\Omega)} \leq$ $C\|u\|_{[T, X](\Omega)}$.

We now prove sufficiency. Since $[T, X](\Omega)$ and $[T, X]^{\mathrm{ri}}(\Omega)$ are isomorphic, there exists a constant $M>0$ such that $\|u\|_{[T, X]^{\mathrm{ri}}(\Omega)} \leq M\|u\|_{[T, X](\Omega)}$. Let $f \in$ $[T, X]_{\mathrm{d}}^{+}$. Choose any $x_{0} \in \Omega$ and $R>0$ such that the open ball $B\left(x_{0}, R\right) \subseteq$ $\Omega$, in which case $C_{n} R^{n} \leq 1\left(C_{n}\right.$ is the measure of the Euclidean unit ball in $\left.\mathbb{R}^{n}\right)$. Define $u: \Omega \rightarrow \mathbb{R}$ by $u(x):=f\left(\left|x-x_{0}\right|^{n} / R^{n}\right)$ on $B\left(x_{0}, R\right)$ and zero elsewhere in $\Omega$. Then $u^{*}(t)=f\left(t /\left(C_{n} R^{n}\right)\right) \chi_{\left[0, C_{n} R^{n}\right]}(t)$, that is, $u^{*}=D_{\beta} f$, with $\beta:=1 /\left(C_{n} R^{n}\right)$. By Proposition 4.1, dilation operators are continuous on $[T, X]$ and so $u^{*} \in[T, X]$, that is, $u \in[T, X](\Omega)$. Then, by our hypothesis, $u \in[T, X]^{\mathrm{ri}}(\Omega)$ and $\|u\|_{[T, X]^{\mathrm{ri}}(\Omega)} \leq M\|u\|_{[T, X](\Omega)}$. That is, $u^{*} \in[T, X]^{\mathrm{ri}}$ and $\left\|u^{*}\right\|_{[T, X]^{\mathrm{ri}}} \leq M\left\|u^{*}\right\|_{[T, X]}$. Consequently, $D_{\beta} f \in[T, X]^{\mathrm{ri}}$ and

$$
\left\|D_{\beta} f\right\|_{[T, X]^{\mathrm{ri}}} \leq M\left\|D_{\beta} f\right\|_{[T, X]} .
$$

Taking into account that dilation operators are bounded on r.i. spaces and that $f=D_{1 / \beta} D_{\beta} f$ (since $\beta \geq 1$ ), we conclude that $f \in[T, X]^{\text {ri }}$. These arguments, together with (4.3) and Proposition 4.1, yield

$$
\|f\|_{[T, X]^{\mathrm{ri}}} \leq M \cdot\left\|D_{1 / \beta}\right\|_{[T, X]^{\mathrm{ri}}} \cdot\left\|D_{\beta}\right\|_{[T, X]} \cdot\|f\|_{[T, X]} .
$$

This completes the proof.

Remark 4.3. For certain classical r.i. spaces $X$, the space $[T, X]^{\text {ri }}$ can be precisely identified. For example, if $X=L^{p}([0,1])$ for $n^{\prime}<p<\infty$, then $[T, X]^{\mathrm{ri}}$ is the Lorentz $L^{p, q}$-space $L^{p_{0}, p}([0,1])$, where $p_{0}:=n p /(n+p)$; when $p=\infty$, we have $\left[T, L^{\infty}\right]^{r i}=L^{n, 1}([0,1])$; and for $1 \leq p \leq n^{\prime}$ we have $[T, X]^{\mathrm{ri}}=L^{1}([0,1])$. For further examples, see [8]. However, in general, the space $[T, X]^{\mathrm{ri}}$ is difficult to identify. Accordingly, when trying to establish (4.2), the following equivalent description of $[T, X]^{\mathrm{ri}}$ can be useful (see [1, Propostion 2.3], for example). Namely, $[T, X]^{\mathrm{ri}}$ is the space of all $f \in[T, X]$ such that $h \in[T, X]$ whenever $h$ is equimeasurable with $f$. Moreover,

$$
\|f \mid\|:=\left\{\sup \|h\|_{[T, X]} \mid h \text { is equimeasurable with } f\right\}
$$

is an equivalent norm in $[T, X]^{\mathrm{ri}}$.

Recall that a function $f$ on $[0,1]$ is said to be equivalent to a decreasing function is there exists a decreasing function $\Phi$ on $[0,1]$ and positive constants $C_{1}$, $C_{2}$ such that $C_{1} \Phi \leq f \leq C_{2} \Phi$. Note that if a function $f$ satisfies $f\left(x_{2}\right) \leq C f\left(x_{1}\right)$ 
for some constant $C>0$ and every $x_{1}<x_{2}$, then it is equivalent to a decreasing function (namely, to the function $\Phi(x):=\sup \{f(t) \mid t \geq x\}$ ).

In order to prove Theorem 1.2, in view of Proposition 4.2, it suffices to check that (4.2) holds.

Proof of Theorem 1.2. Let $\Phi$ be a decreasing function such that

$$
C_{1} \Phi(t) \leq \varphi(t) / t^{1 / n^{\prime}} \leq C_{2} \Phi(t), \quad \text { for some } C_{1}, C_{2}>0 .
$$

Let $f \in\left[T, \Lambda_{\varphi}\right]_{\mathrm{d}}^{+}$. Then $f=f^{*}$ and $T f^{*} \in \Lambda_{\varphi}$. Let $h$ be any function equimeasurable with $f$. Since $|h| \geq 0$, it is clear from (1.3) that $T|h|$ is decreasing. Applying (2.1) and Hardy's lemma (since $\Phi$ is decreasing) we get via Fubini's theorem that

$$
\begin{aligned}
\|h\|_{\left[T, \Lambda_{\varphi}\right]} & =\|T|h|\|_{\Lambda_{\varphi}}=\int_{0}^{1} T|h(s)| \varphi^{\prime}(s) \mathrm{d} s \\
& =\int_{0}^{1} \int_{S}^{1}|h(t)| t^{-1 / n^{\prime}} \mathrm{d} t \varphi^{\prime}(s) \mathrm{d} s=\int_{0}^{1}|h(t)| t^{-1 / n^{\prime}} \varphi(t) \mathrm{d} t \\
& \leq C_{2} \int_{0}^{1}|h(t)| \Phi(t) \mathrm{d} t \leq C_{2} \int_{0}^{1} f^{*}(t) \Phi(t) \mathrm{d} t \\
& \leq \frac{C_{2}}{C_{1}} \int_{0}^{1} f^{*}(t) t^{-1 / n^{\prime}} \varphi(t) \mathrm{d} t=\frac{C_{2}}{C_{1}} \int_{0}^{1} T f^{*}(s) \varphi^{\prime}(s) \mathrm{d} s \\
& =\frac{C_{2}}{C_{1}}\left\|T f^{*}\right\|_{\Lambda_{\varphi}}=\frac{C_{2}}{C_{1}}\left\|f^{*}\right\|_{\left[T, \Lambda_{\varphi}\right]}=\frac{C_{2}}{C_{1}}\|f\|_{\left[T, \Lambda_{\varphi}\right]} .
\end{aligned}
$$

Thus $h \in\left[T, \Lambda_{\varphi}\right]$ and $\|h\|_{\left[T, \Lambda_{\varphi}\right]} \leq\left(C_{2} / C_{1}\right)\|f\|_{\left[T, \Lambda_{\varphi}\right]}$. From Remark 4.3 it follows that $f \in\left[T, \Lambda_{\varphi}\right]^{\mathrm{ri}}$ and $\|f\|_{\left[T, \Lambda_{\varphi}\right]^{\mathrm{ri}}} \leq\left(C_{2} / C_{1}\right)\|f\|_{\left[T, \Lambda_{\varphi}\right]}$. The result then follows from Proposition 4.2.

Theorem 1.3 will follow from a more general result; see Theorem 4.5 below. Recall that the associate space $X^{\prime}$ of $X$, also r.i. over $[0,1]$, is the space of all functions $g$ on $[0,1]$ for which $f g \in L^{1}([0,1])$, for every $f \in X$, [2, 2.4.2]. The classical Hardy operator $H$ is defined by

$$
H f: t \mapsto \frac{1}{t} \int_{0}^{t} f(s) \mathrm{d} s, \quad t \in(0,1], f \in \mathcal{M}^{+} .
$$

Lemma 4.4. Let $X$ be an r.i. space over $[0,1]$ for which there exists a constant $C>0$ such that

$$
\left\|t^{-1 / n}\left(s^{1 / n} H g(s)\right)^{*}(t)\right\|_{X^{\prime}} \leq C \cdot\|g\|_{X^{\prime}}, \quad g \in X^{\prime} .
$$


Then there exists a constant $M>0$ such that, whenever $f_{1}, f_{2}$ are nonnegative functions on $[0,1]$ satisfying $f_{1} \prec f_{2}$ and $T f_{2}^{*} \in X$, the function $T f_{1} \in X$ and

$$
\left\|T f_{1}\right\|_{X} \leq M\left\|T f_{2}^{*}\right\|_{X} .
$$

Proof. Let $f_{1}, f_{2}$ be nonnegative functions satisfying the stated conditions. Fix $g \in X^{\prime}$. For a decreasing function $h \geq 0$ on $[0,1]$ also $s^{-1 / n^{\prime}} h(s)$ is decreasing. Then, for each $t \in[0,1]$, we have

$$
\begin{aligned}
2\left(D_{1 / 2} T h\right)(t)=2 T h\left(\frac{t}{2}\right) & =2 \int_{t / 2}^{1} h(s) s^{-1 / n^{\prime}} \mathrm{d} s \\
& \geq 2 \int_{t / 2}^{t} h(s) s^{-1 / n^{\prime}} \mathrm{d} s \geq t^{1 / n} h(t) .
\end{aligned}
$$

Applying (2.1), Hardy's lemma, the previous inequality and the hypothesis, we get (via Fubini's theorem)

$$
\begin{aligned}
\int_{0}^{1} T f_{1}(x) g(x) \mathrm{d} x & =\int_{0}^{1} f_{1}(t) t^{-1 / n^{\prime}} \int_{0}^{t} g(x) \mathrm{d} x \mathrm{~d} t \\
& =\int_{0}^{1} f_{1}(t) t^{1 / n} H g(t) \mathrm{d} t \leq \int_{0}^{1} f_{1}^{*}(t)\left(s^{1 / n} H g(s)\right)^{*}(t) \mathrm{d} t \\
& \leq \int_{0}^{1} f_{2}^{*}(t)\left(s^{1 / n} H g(s)\right)^{*}(t) \mathrm{d} t \\
& \leq \int_{0}^{1} \frac{2}{t^{1 / n}} D_{1 / 2} T f_{2}^{*}(t)\left(s^{1 / n} H g(s)\right)^{*}(t) \mathrm{d} t \\
& \leq 2\left\|D_{1 / 2} T f_{2}^{*}(t)\right\|_{X} \cdot\left\|t^{-1 / n}\left(s^{1 / n} H g(s)\right)^{*}(t)\right\|_{X^{\prime}} \\
& \leq 2 C\left\|D_{1 / 2}\right\|_{X}\left\|T f_{2}^{*}\right\|_{X}\|g\|_{X^{\prime}} .
\end{aligned}
$$

Taking the supremum over $g$ in the unit ball of $X^{\prime}$, we get $T f_{1} \in X$ and $\left\|T f_{1}\right\|_{X} \leq$ $M\left\|T f_{2}^{*}\right\|_{X}$.

Theorem 4.5. Let $X$ be an r.i. space over $[0,1]$ satisfying inequality (4.4). Then the optimal Sobolev imbedding (1.7) exists for X but, it is not a further extension of the optimal r.i. Sobolev imbedding (1.5).

Proof. Let $f \in[T, X]_{\mathrm{d}}^{+}$. Then $f=f^{*}$ and $T f^{*} \in X$. Let $h$ be any function equimeasurable with $f$. Then $|h| \prec f^{*}$. Thus, applying Lemma 4.4, we obtain that $T|h| \in X$ and $\|T|h|\|_{X} \leq M\|T f\|_{X}$, that is, $h \in[T, X]$ and $\|h\|_{[T, X]} \leq$ $M\|f\|_{[T, X]}$, for a certain constant $M>0$. From Remark 4.3, it follows that $f \in$ $[T, X]^{\mathrm{ri}}$ and $\|f\|_{[T, X]^{\mathrm{ri}}} \leq M\|f\|_{[T, X]}$. The result then follows from Proposition 4.2. 
In order to deduce Theorem 1.3 from Theorem 4.5 we need some auxiliary results. Recall, if $\varphi$ is a positive function defined on $[0,1]$, then its lower dilation index, [12, II.1.2], is defined by

$$
\gamma_{\varphi}:=\lim _{t \rightarrow 0} \frac{\log \sup _{0<s<1}(\varphi(s t) / \varphi(s))}{\log t} .
$$

Lemma 4.6 ([5, Lemma 5.6]). Let $\alpha>0$. Let $\varphi$ be an increasing, concave function with $\varphi(0)=0$ and $\gamma_{\varphi}>\alpha$. Then there exists a constant $C>0$, depending only on $\alpha$ and $\varphi$, such that

$$
\int_{0}^{1}\left(s^{\alpha} g(s)\right)^{*}(t) t^{-\alpha} \varphi^{\prime}(t) \mathrm{d} t \leq C \int_{0}^{1} g(t) \varphi^{\prime}(t) \mathrm{d} t,
$$

for every decreasing function $g$ on $[0,1]$.

The following result will later be seen to be self improving.

Lemma 4.7. Inequality (4.4) is satisfied for $X=L^{p, q}([0,1])$ whenever $n^{\prime}<$ $p<\infty$ and $p \leq q$.

Proof. For $X=L^{p, q}([0,1])$ the associate space is $X^{\prime}=L^{p^{\prime}, q^{\prime}}([0,1])$. Fix $g \in X^{\prime}$. Let $\alpha=q^{\prime} / n$ and define $\varphi^{\prime}(t)=\left(p^{\prime} / q^{\prime}\right) t^{q^{\prime}} / p^{\prime}$. In order to apply Lemma 4.6 we need $\varphi$ to be concave, that is, $\left(q^{\prime} / p^{\prime}\right)-1 \leq 0$, and also $\gamma_{\varphi}>q^{\prime} / n$, that is, $q^{\prime} / p^{\prime}>q^{\prime} / n$. Hence, for $q^{\prime} \geq p^{\prime}$ and $n>p^{\prime}$, that is, $n^{\prime}<p<\infty$ and $p \leq q$, we can apply the lemma (together with $|H g| \leq H g^{*}$ ) to get

$$
\begin{aligned}
\left\|t^{-1 / n}\left(s^{1 / n} H g(s)\right)^{*}(t)\right\|_{p^{\prime}, q^{\prime}}^{q^{\prime}} & \leq\left\|t^{-1 / n}\left(s^{1 / n} H g^{*}(s)\right)^{*}(t)\right\|_{p^{\prime}, q^{\prime}}^{q^{\prime}} \\
& =\int_{0}^{1}\left(t^{1 / p^{\prime}} t^{-1 / n}\left(s^{1 / n} H g^{*}(s)\right)^{*}(t)\right)^{q^{\prime}} \frac{\mathrm{d} t}{t} \\
& =\int_{0}^{1} t^{-q^{\prime} / n}\left(s^{q^{\prime} / n}\left(H g^{*}(s)\right)^{q^{\prime}}\right)^{*}(t) t^{\left(q^{\prime} / p^{\prime}\right)-1} \mathrm{~d} t \\
& \leq C \int_{0}^{1}\left(H g^{*}\right)^{q^{\prime}}(t) t^{\left(q^{\prime} / p^{\prime}\right)-1} \mathrm{~d} t \\
& =C\left\|H g^{*}\right\|_{p^{\prime}, q^{\prime}}^{q^{\prime}} .
\end{aligned}
$$

For the boundedness of the Hardy operator on $L^{p^{\prime}, q^{\prime}}([0,1])$ we need $p^{\prime}>1$, that is, $p<\infty$. Under these conditions (4.4) holds.

Recall that a mapping $Q$ from a Banach space into a Banach lattice is quasi-linear if:

(a) $|Q(a x)|=|a| \cdot|Q x|$, for $a \in \mathbb{R}$; and 
(b) there exists $C>0$ so that $\left|Q\left(x_{1}+x_{2}\right)\right| \leq C\left(\left|Q x_{1}\right|+\left|Q x_{2}\right|\right)$.

The quasi-linear operator $Q$ is said to be bounded if there exists $M>0$ such that $\|Q x\| \leq M\|x\|$ (see $[13,2 . a .9])$ and of strong type $(p, q)$ if it is bounded from $L^{p}$ to $L^{q}$; see $[13,2$. b.10].

Proof of Theorem 1.3. First note that in (4.4) we can replace $g$ with $|g|$, since $\||g|\|_{X^{\prime}}=\|g\|_{X^{\prime}}$ and $|H g| \leq H|g|$. Consider the operator

$$
Q: g(t) \longmapsto t^{-1 / n}\left(s^{1 / n} H|g|(s)\right)^{*}(t) .
$$

We check that $Q$ is quasi-linear. Standard properties of decreasing rearrangements show that $|Q(a g)|=|a| \cdot|Q g|$ for $a \in \mathbb{R}$. Taking into account (2.3), that $D_{\alpha}\left(f^{*}\right)=\left(D_{\alpha} f\right)^{*}$ for any dilation operator $D_{\alpha},[2$, p.148], and that $H|g|(s / 2) \leq$ $2 H|g|(s)$, we have

$$
\begin{aligned}
\mid Q\left(g_{1}\right. & \left.+g_{2}\right)(t) \mid=t^{-1 / n}\left(s^{1 / n} H\left|g_{1}+g_{2}\right|(s)\right)^{*}(t) \\
& \leq t^{-1 / n}\left(s^{1 / n} H\left|g_{1}\right|(s)+s^{1 / n} H\left|g_{2}\right|(s)\right)^{*}(t) \\
& \leq t^{-1 / n}\left(s^{1 / n} H\left|g_{1}\right|(s)\right)^{*}\left(\frac{t}{2}\right)+t^{-1 / n}\left(s^{1 / n} H\left|g_{2}\right|(s)\right)^{*}\left(\frac{t}{2}\right) \\
& =t^{-1 / n}\left(\left(\frac{s}{2}\right)^{1 / n} H\left|g_{1}\right|\left(\frac{s}{2}\right)\right)^{*}(t)+t^{-1 / n}\left(\left(\frac{s}{2}\right)^{1 / n} H\left|g_{2}\right|\left(\frac{s}{2}\right)\right)^{*} \\
& \leq 2^{1 / n^{\prime}} t^{-1 / n}\left(s^{1 / n} H\left|g_{1}\right|(s)\right)^{*}(t)+2^{1 / n^{\prime}} t^{-1 / n}\left(s^{1 / n} H\left|g_{2}\right|(s)\right)^{*}(t) \\
& =2^{1 / n^{\prime}}\left(Q\left(g_{1}\right)(t)+Q\left(g_{2}\right)(t)\right) .
\end{aligned}
$$

From Lemma 4.7, the inequality (4.4) holds for $X=L^{p, q}([0,1])$ provided that $n^{\prime}<p<\infty$ and $p \leq q$, that is, for $X^{\prime}=L^{p^{\prime}, q^{\prime}}([0,1])$ with $1<p^{\prime}<n$ and $q^{\prime} \leq p^{\prime}$. In particular, (4.4) holds for all $X^{\prime}=L^{p^{\prime}}([0,1])$ with $1<p^{\prime}<n$. This means precisely that the quasi-linear operator $Q$ is of strong type $\left(p^{\prime}, p^{\prime}\right)$. Choose any $1<p_{1}<p_{2}<n$. Since $Q$ is of strong types $\left(p_{1}, p_{1}\right)$ and $\left(p_{2}, p_{2}\right)$, by a theorem of Boyd (see [13, Theorem 2.b.11 \& Remark 1, p.147]) it is known that $Q$ is bounded from $Z$ to $Z$ for every r.i. space $Z$ whose Boyd indices satisfy $1 / p_{2}<\underline{\alpha}_{Z} \leq \bar{\alpha}_{Z}<1 / p_{1}$. But, this is the case for every choice of $1<p_{1}<$ $p_{2}<n$. It follows that $Q$ is bounded from $Z$ to $Z$ for every r.i. space $Z$ satisfying $1 / n<\underline{\alpha}_{Z} \leq \bar{\alpha}_{Z}<1$. Setting $Z=X^{\prime}$ and taking into account that $\underline{\alpha}_{X^{\prime}}=1-\bar{\alpha}_{X}$ and $\bar{\alpha}_{X^{\prime}}=1-\underline{\alpha}_{X},[2,3.5 .13]$, it follows that (4.4) is satisfied for every r.i. space $X$ satisfying $0<\underline{\alpha}_{X} \leq \bar{\alpha}_{X}<1 / n^{\prime}$.

Proposition 4.2 shows that the r.i. q-B.f.s. $[T, X](\Omega)$ is isomorphic to an r.i. B.f.s. (i.e., is normable) whenever $[T, X]_{\mathrm{d}}^{+} \subset[T, X]^{\mathrm{ri}}$ and (4.2) is satisfied. This forms the basis of the proof of our final result (which covers all the spaces $X$ occurring in this section); compare with Proposition 3.2. 
Proposition 4.8. Let $X$ be an r.i. space over $[0,1]$. The space $[T, X](\Omega)$ is isomorphic to an r.i. Banach function space (i.e., it is normable) in each of the following situations:

(a) $X=\Lambda_{\varphi}$ is a Lorentz $\Lambda$-space such that $\varphi(t) / t^{1 / n^{\prime}}$ is equivalent to a decreasing function.

(b) $X$ satisfies the inequality (4.4).

(c) The Boyd indices of $X$ satisfy $0<\underline{\alpha}_{X} \leq \bar{\alpha}_{X}<1 / n^{\prime}$.

Proof. (a) In the proof of Theorem 1.2 it was shown, under the given condition on $\varphi$, that $[T, X]_{\mathrm{d}}^{+} \subset[T, X]^{\mathrm{ri}}$ and (4.2) is satisfied. By Proposition 4.2, $[T, X](\Omega)$ is then isomorphic to the r.i. B.f.s. $[T, X]^{\mathrm{ri}}(\Omega)$.

(b) According to the proof of Theorem 4.5, the inequality (4.4) also implies that $[T, X]_{\mathrm{d}}^{+} \subset[T, X]^{\mathrm{ri}}$ and that (4.2) is satisfied.

(c) An examination of the proof of Theorem 1.3 shows that (4.4) follows from the assumptions on the Boyd indices of $X$ and so (b) applies.

Acknowledgements The authors acknowledge the support of the Maximilian Bickhoff-Universitätsstiftung (Eichstätt, Germany), D.G.I. \#BFM2003-06335C03-01 (Spain) and FQM 262 (Junta de Andalucía).

\section{REFERENCES}

[1] Serguei V. Astashin and Guillermo P. Curbera, Symmetric kernel of Rademacher multiplicator spaces, J. Funct. Anal. 226 (2005), 173-192,

http://dx.doi.org/10.1016/j.jfa.2005.01.014. MR 2158179 (2006b:46032)

[2] Colin Bennett and Robert Sharpley, Interpolation of Operators, Pure and Applied Mathematics, vol. 129, Academic Press Inc., Boston, MA, 1988, ISBN 0-12-088730-4. MR 928802 (89e:46001)

[3] Andrea Cianchi and Luboš Pick, Sobolev embeddings into BMO,VMO, and $L_{\infty}$, Ark. Mat. 36 (1998), 317-340, http://dx.doi.org/10.1007/BF02384772. MR 1650446 (99k:46052)

[4] Guillermo P. Curbera and Werner J. Ricker, Optimal domains for kernel operators via interpolation, Math. Nachr. 244 (2002), 47-63,

http://dx.doi.org/10.1002/1522-2616(200210)244:1;47::AID-MANA47;3.0.CO;2-B. $\quad$ MR 1928916 (2003g:47045)

[5] _ Optimal domains for the kernel operator associated with Sobolev's inequality, Studia Math. 158 (2003), 131-152. MR 2013736 (2005b:46068)

[6] _ Corrigenda to: "Optimal domains for the kernel operator associated with Sobolev's inequality” [Studia Math. 158 (2003), no. 2, 131-152; MR2013736], Studia Math. 170 (2005), 217218. MR 2184107 (2007b:46047)

[7] _ Banach lattices with the Fatou property and optimal domains of kernel operators, Indag. Math. (N.S.) 17 (2006), 187-204, http://dx.doi.org/10.1016/S0019-3577(06)80015-7.

[8] _ Compactness properties of Sobolev imbeddings for rearrangement invariant norms, Trans. Amer. Math. Soc. 359 (2007), 1471-1484 (electronic), http://dx.doi.org/10.1090/S0002-9947-06-04203-6. MR 2272134 
[9] David E. Edmunds, Ron Kerman, and Luboš Pick, Optimal Sobolev imbeddings involving rearrangement-invariant quasinorms, J. Funct. Anal. 170 (2000), 307-355, http://dx.doi.org/10.1006/jfan.1999.3508. MR 1740655 (2000m:46070)

[10] Nigel J. Kalton, N.T. Peck, and James W. Roberts, An F-space Sampler, London Mathematical Society Lecture Note Series, vol. 89, Cambridge University Press, Cambridge, 1984, ISBN 0-521-27585-7. MR 808777 (87c:46002)

[11] GotTfried KÖTHe, Topological vector spaces. I, Translated from the German by D. J. H. Garling. Die Grundlehren der mathematischen Wissenschaften, Band 159, Springer-Verlag, New York, 1969. MR 0248498 (40 \#1750)

[12] S.G. KReĬN, Yu. İ. Petunīn, and E.M. SEMËNOV, Interpolation of Linear Operators, Translations of Mathematical Monographs, vol. 54, American Mathematical Society, Providence, R.I., 1982, ISBN 0-8218-4505-7, Translated from the Russian by J. Szúcs. MR 649411 (84j:46103)

[13] Joram Lindenstrauss and Lior TZAFriri, Classical Banach spaces. II. Function Spaces, Ergebnisse der Mathematik und ihrer Grenzgebiete [Results in Mathematics and Related Areas], vol. 97, Springer-Verlag, Berlin, 1979, ISBN 3-540-08888-1. MR 540367 (81c:46001)

[14] AdriaAn CORnElis ZAANEN, Integration, Completely revised edition of An introduction to the theory of integration, North-Holland Publishing Co., Amsterdam; Interscience Publishers John Wiley \& Sons, Inc., New York, 1967. MR 0222234 (36 \#5286)

Guillermo P. Curbera:

Facultad de Matemáticas

Universidad de Sevilla

Aptdo. 1160, Sevilla 41080, Spain.

E-MAIL: curbera@us.es

URL: http://euler.us.es/ curbera/

WERNER J. RICKER:

Math.-Geogr. Fakultät

Katholische Universität Eichstätt-Ingolstadt

D-85072 Eichstätt, Germany.

E-MAIL: werner.ricker@ku-eichstaett.de

URL: http://www.ku-eichstaett.de/Fakultaeten/MGF/Mathematik/Lehrstuhl.Analysis.de

KEY WORDS AND PHRASES: Sobolev imbeddings, rearrangement invariant spaces, Banach function spaces, optimal domains.

2000 Mathematics Subject Classification: 46E35, 46E30 (47G10).

Received: December 21st, 2005; revised: March 29th, 2006.

Article electronically published on May 30th, 2007. 